\title{
Impact of low-energy photons on the characteristics of prompt fission $\gamma$-ray spectra
}

\author{
A. Oberstedt, ${ }^{1,2}$ R. Billnert, ${ }^{1,3}$ F.-J. Hambsch, ${ }^{3}$ and S. Oberstedt ${ }^{3, *}$ \\ ${ }^{1}$ Fundamental Fysik, Chalmers Tekniska Högskola, S-41296 Göteborg, Sweden \\ ${ }^{2}$ OSSOLUTIONS Consulting, S-70353 Örebro, Sweden \\ ${ }^{3}$ European Commission, DG Joint Research Centre (IRMM), B-2440 Geel, Belgium \\ (Received 9 May 2014; revised manuscript received 23 March 2015; published 20 July 2015)
}

\begin{abstract}
In this paper we report on a new study of prompt $\gamma$-rays from the spontaneous fission of ${ }^{252} \mathrm{Cf}$. Photons were measured in coincidence with fission fragments by employing four different lanthanide halide scintillation detectors. Together with results from a previous work of ours, we determined characteristic parameters with high precision, such as the average $\gamma$-ray multiplicity $\bar{v}_{\gamma}=(8.29 \pm 0.13)$, the average energy per photon $\epsilon_{\gamma}=(0.80 \pm$ $0.02) \mathrm{MeV}$, and the total $\gamma$-ray energy release per fission $E_{\gamma \text {,tot }}=(6.65 \pm 0.10) \mathrm{MeV}$. The excellent agreement between the individual results obtained in all six measurements proves the good repeatability of the applied experimental technique. The impact of low-energy photons, i.e., below $500 \mathrm{keV}$, on prompt fission $\gamma$-ray spectra characteristics has been investigated as well by comparing our results with those taken with the DANCE detector system, which appears to suffer from absorption effects in the low-energy region. Correction factors for this effect were estimated, giving results comparable to ours as well as to historical ones. From this we demonstrate that the different techniques of determining the average $\gamma$-ray multiplicity, either from a properly measured and normalized spectrum or a measured multiplicity distribution, give equivalent and consistent results.
\end{abstract}

DOI: 10.1103/PhysRevC.92.014618

PACS number(s): 24.75.+i, 07.85.Nc, 25.85.Ca, 29.30.Kv

\section{INTRODUCTION}

In a recent paper results were presented from precise measurements of prompt fission $\gamma$-ray spectra (PFGS) from the reaction $\mathrm{n}_{t h}+{ }^{235} \mathrm{U}$ [1]. Their characteristics in terms of average $\gamma$-ray multiplicity $\bar{\nu}_{\gamma}$, average energy per photon and total $\gamma$-ray energy release per fission, $\epsilon_{\gamma}$ and $E_{\gamma, \text { tot }}$, respectively, were determined for $\gamma$-rays with energies between 0.1 and 6.0 MeV. Since the experimental results were compared with both previous measurements [2-4] and recent Monte Carlo Hauser-Feshbach calculations [5-8], which cover an energy range up to $10 \mathrm{MeV}$ and more, the impact of $\gamma$-rays with energies above $6 \mathrm{MeV}$ was investigated. This was done by fitting a measured spectrum between 3 and $6 \mathrm{MeV}$ with an exponential function, extrapolating it to $10 \mathrm{MeV}$ and integrating between 6 and $10 \mathrm{MeV}$. A detailed description of this procedure is given in Ref. [1]. As a result it could be estimated that the contribution of these high energetic photons on the total $\gamma$-ray energy released per fission is at most of the order of the uncertainty of its mean value.

In the present work, in contrast, we investigated the impact of the low-energy part of PFGS on their characteristics. The study was motivated by the fact that experimental results obtained by our collaboration agree quite well with corresponding historical experimental as well as recent theoretical values, but disagree considerably with those taken with the DANCE detector system. This has already been shown for the reactions ${ }^{252} \mathrm{Cf}(\mathrm{sf})[9],{ }^{235} \mathrm{U}\left(n_{\text {th }}, f\right)[1]$, and ${ }^{241} \mathrm{Pu}\left(n_{\text {th }}, f\right)$

\section{*Stephan.OBERSTEDT@ec.europa.eu}

Published by the American Physical Society under the terms of the Creative Commons Attribution 3.0 License. Further distribution of this work must maintain attribution to the author(s) and the published article's title, journal citation, and DOI.
[10], in particular for $\epsilon_{\gamma}$ and $E_{\gamma \text {,tot }}$. These discrepancies were associated with absorption effects in DANCE for photons in the low-energy regime [9], which becomes rather obvious by comparing the shapes of the PFGS below $500 \mathrm{keV}$. In order to assess this effect numerically and eventually offer a possibility to correct for it, we revisited our measurements of PFGS from the spontaneous fission of ${ }^{252} \mathrm{Cf}(\mathrm{sf})$. In addition to our previous results published in Ref. [9], obtained with a $\mathrm{LaBr}_{3}: \mathrm{Ce}$ and a $\mathrm{CeBr}_{3}$ scintillation detector, respectively, we performed and analyzed four more measurements employing three other detectors. The results are given below.

\section{EXPERIMENTS AND RESULTS}

The new measurements were performed by putting a ${ }^{252} \mathrm{Cf}$ source inside a cylindrical vacuum chamber. The fast fission trigger was provided by an artificial polycrystalline chemical vapor deposited (pcCVD) diamond detector, whose active area was $1 \mathrm{~cm} \times 1 \mathrm{~cm}$, and which was mounted directly above the ${ }^{252} \mathrm{Cf}$ sample. The properties of the artificial diamond detector are described in Ref. [12]. In one of the measurements a coaxial cerium-doped lanthanum chloride $\left(\mathrm{LaCl}_{3}: \mathrm{Ce}\right)$ scintillation detector of size 1.5 in. $\times 1.5$ in. was placed at a distance of $37 \mathrm{~cm}$ from the fission source in order to measure photons in coincidence with fission fragments. More detailed information about the experimental setup and employed electronics may be found in Ref. [11], where also results from the characterization of the used scintillation detector is given. In other measurements, coaxial ceriumdoped lanthanum bromide $\left(\mathrm{LaBr}_{3}: \mathrm{Ce}\right)$ scintillation detectors of size 2 in. $\times 2$ in. (two times) and 3 in. $\times 3$ in. were used in a similar setup. They have already been used in previous experiments (cf. Refs. [1,9,10], where also references to information about their properties are given). 
Here too, the distance between detector and ${ }^{252} \mathrm{Cf}$ sample was $40 \mathrm{~cm}$ for the smaller detectors and $60 \mathrm{~cm}$ for the bigger one. All detectors were placed perpendicular to the symmetry axis of the ionization chamber in the same plane as the fission source. Hence, they all have in common that their covered solid angle is about $4 \pi / 1000$, indicating that the probability of multiple photon events decreases for each photon by a factor of $1 / 1000$. The observed coincidence timing resolution at $E_{\gamma}=$ $200 \mathrm{keV}$ was about $1 \mathrm{~ns}$ for $\mathrm{LaBr}_{3}: \mathrm{Ce}$ and $1.1 \mathrm{~ns}$ for $\mathrm{LaCl}_{3}: \mathrm{Ce}$ detectors in conjunction with a diamond detector, which is better than obtained with a Frisch grid ionization chamber used as fission trigger [10]. Fission $\gamma$-rays were selected within \pm 3 ns relative to the prompt peak in the time-of-flight spectrum; see Ref. [10] for comparison.

After obtaining a background-subtracted prompt fission $\gamma$-ray spectrum, the response function of the respective scintillation detectors must be unfolded in order to extract the real emission spectra. These response functions were determined by means of Monte Carlo simulations with the computer code PENELOPE2011 [13], where experimental conditions, like, e.g., acceptance of the corresponding detectors, were taken into account. The measured spectra were then reproduced with the results from the simulations and the actually emitted prompt fission $\gamma$-ray spectrum was deduced according to a technique that has been described in Ref. [9] and already applied successfully on seven measurements involving five different lanthanide halide detectors [1,9] prior to this work.

When both measuring time and effective activity of the sample are known, the adjustment of the integral simulated spectrum to the experimental one gives automatically the properly normalized emission spectrum in units per $\mathrm{MeV}$ and fission. Hence, integrating over the entire energy spectrum gives the number of $\gamma$-rays per fission, i.e., the multiplicity. The effective activity depends on the total activity, the spatial extension of the radioactive source, and the coverage of the diamond detector, which is important, since it determines the fission trigger rate. This, however, was not very well known in the measurement with the $\mathrm{LaCl}_{3}$ :Ce detector. Hence, the emission spectrum for that detector obtained in this work was adjusted in height to a corresponding spectrum taken previously with a $\mathrm{LaBr}_{3}$ :Ce detector [9]. This adjustment was performed by normalizing the integral spectrum to the reference spectrum within the energy range between 2 and $6 \mathrm{MeV}$.

The resulting emission spectra from this work are shown in Fig. 1(a), where the different detectors in use are indicated. For the sake of clarity, error bars are shown only for one detector (Q489). Within these uncertainties, the agreement between all four spectra is very good over the entire energy range. In Fig. 1(b) one of the spectra obtained in this work is compared to results from different theoretical efforts. As mentioned above, new and advanced computer codes are able to simulate prompt neutron and $\gamma$-ray emission from primary fission fragments by a Monte Carlo approach together with a full Hauser-Feshbach calculation, developed independently at CEA Cadarache $[5,6]$ and by a Los Alamos/New York collaboration [7,8]. Both theoretical models provided already predictions for PFGS characteristics for the system $\mathrm{n}_{t h}+{ }^{235} \mathrm{U}$, which are in good agreement with recent experimental results [1]. Both research groups supplied us also with results from new and improved
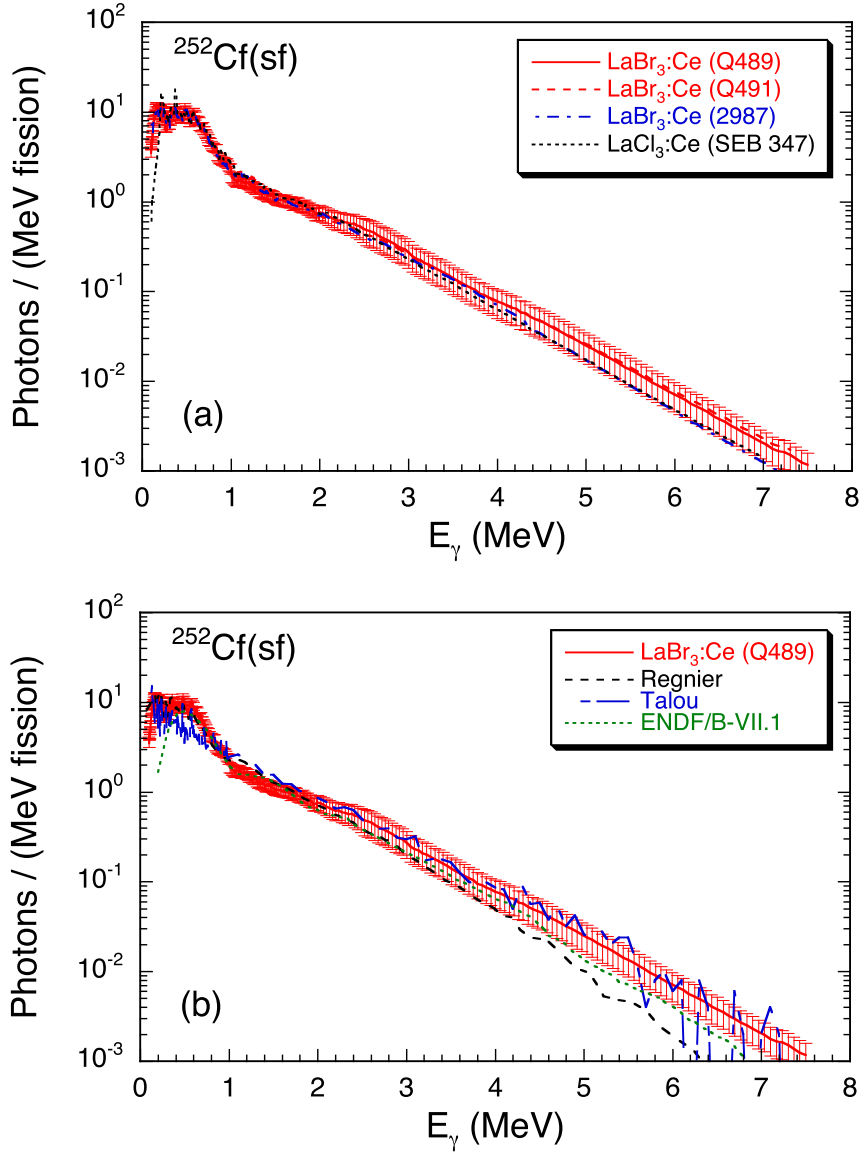

FIG. 1. (Color online) (a) Unfolded prompt fission $\gamma$-ray emission spectra from the spontaneous fission of ${ }^{252} \mathrm{Cf}$, taken with four different lanthanide halide scintillation detectors: three $\mathrm{LaBr}_{3}: \mathrm{Ce}$ detectors and the $\mathrm{LaCl}_{3}$ :Ce detector used in this work (see text for details). (b) Comparison between one of the experimental spectra from this work with the ones of model calculations from Refs. [14,15] and data from the evaluated library ENDF/B-VII.1 [16]. Apart from the energy range above $4 \mathrm{MeV}$, the slopes of all spectra are in rather good agreement, i.e., within the error bars from our measurement, which for the sake of clarity are shown for the first mentioned detector only.

calculations for the spontaneous fission of ${ }^{252} \mathrm{Cf}$, which include both PFGS characteristics and calculated spectra [14,15]. The latter are depicted in the lower part of Fig. 1, together with information from the recently updated evaluated data base ENDF/B-VII.1 [16]. The agreement between all data is rather good, despite differences with respect to the slopes above $4 \mathrm{MeV}$ and the fact that the evaluation underestimates the photon yield at energies below $400 \mathrm{keV}$. Below the characteristic parameters describing PFGS are determined and compared as well.

For that, the integral and first moment of the measured energy distributions are calculated, representing the average photon multiplicity and the total photon energy, respectively. The average energy per photon is then given by the ratio of average total photon energy and average multiplicity. The uncertainties of the integrated values contain statistical uncertainties in the measured spectra as well as contributions 
TABLE I. Summary of prompt $\gamma$-ray characteristics for the spontaneous fission of ${ }^{252} \mathrm{Cf}$. Experimental results from this work for the $\gamma$-ray multiplicity $\bar{v}_{\gamma}$, the average energy $\epsilon_{\gamma}$, and the total energy $E_{\gamma \text {,tot }}$ are given and the covered energy range is indicated. The results from our previous work [9] are given as well and averaged values for our six measurements are presented. They are compared to previously obtained experimental results from Refs. [2,17,18] as well as to corresponding numbers from the evaluated nuclear data files in ENDF/B-VII.1 [16] and from calculations from Refs. [5,6,8,14,15]. The results denoted by* are calculated on the basis of an adjustment to a result from Ref. [9] (see text for details). The bottom line corresponds to modified values from Ref. [17] according to a procedure described in Sec. III.

\begin{tabular}{|c|c|c|c|c|c|c|}
\hline Results & Detector & $\begin{array}{l}\text { Diameter } \times \text { length } \\
\quad(\text { in. } \times \text { in. })\end{array}$ & $\begin{array}{c}\bar{v}_{\gamma} \\
\text { (per fission) }\end{array}$ & $\begin{array}{c}\epsilon_{\gamma} \\
(\mathrm{MeV})\end{array}$ & $\begin{array}{l}\mathrm{E}_{\gamma, \text { tot }} \\
(\mathrm{MeV})\end{array}$ & $\begin{array}{c}\text { Energy range } \\
(\mathrm{MeV})\end{array}$ \\
\hline This work & $\mathrm{LaBr}_{3}: \mathrm{Ce}(\mathrm{Q} 489)$ & $2 \times 2$ & $8.29 \pm 0.07$ & $0.81 \pm 0.01$ & $6.74 \pm 0.09$ & $0.1-6.4$ \\
\hline This work & $\mathrm{LaBr}_{3}: \mathrm{Ce}(2987)$ & $3 \times 3$ & $8.28 \pm 0.07$ & $0.79 \pm 0.01$ & $6.51 \pm 0.07$ & $0.1-7.7$ \\
\hline This work & $\mathrm{LaCl}_{3}: \mathrm{Ce}(\mathrm{SEB} 347)$ & $1.5 \times 1.5$ & $8.28 \pm 0.44$ & $0.82 \pm 0.05$ & $6.81 \pm 0.14$ & $0.1-6.0$ \\
\hline Billnert et al. [9] & $\mathrm{LaBr}_{3}: \mathrm{Ce}(\mathrm{Q} 489)$ & $2 \times 2$ & $8.28 \pm 0.08$ & $0.80 \pm 0.01$ & $6.64 \pm 0.10$ & $0.1-7.2$ \\
\hline This work & \multicolumn{2}{|c|}{ Averaged values } & $8.29 \pm 0.13$ & $0.80 \pm 0.02$ & $6.65 \pm 0.10$ & $>0.1$ \\
\hline Chyzh et al. [17] & \multicolumn{2}{|c|}{ DANCE [19] } & $8.14 \pm 0.40$ & $0.94 \pm 0.05$ & $7.65 \pm 0.55$ & $0.15-9.3$ \\
\hline Verbinski et al. [2] & $\mathrm{NaI}$ & $2.3 \times 6$ & $7.80 \pm 0.30$ & $0.88 \pm 0.04$ & $6.84 \pm 0.30$ & $0.14-10.0$ \\
\hline Pleasonton et al. [18] & $\mathrm{NaI}$ & $5 \times 4$ & $8.32 \pm 0.40$ & $0.85 \pm 0.06$ & $7.06 \pm 0.35$ & $>0.085$ \\
\hline ENDF/B-VII.1 [16] & \multicolumn{2}{|c|}{ Evaluation } & 8.58 & 0.78 & 6.71 & $0.1-10.0$ \\
\hline Talou [15] & \multicolumn{2}{|c|}{ Calculation } & 7.96 & 0.86 & 6.85 & $0.1-10.0$ \\
\hline This work & \multicolumn{2}{|c|}{ Modified results from Chyzh et al. [17] } & $8.40 \pm 0.81$ & $0.76 \pm 0.08$ & $6.38 \pm 0.91$ & $0.1-9.3$ \\
\hline
\end{tabular}

from the Monte Carlo simulations of the response function, the unfolding procedure, and from all other data treatment. The relative contributions of statistical and systematic uncertainties are given in Table II. The adjustment of the spectrum taken with the $\mathrm{LaCl}_{3}$ :Ce detector introduced an additional uncertainty, which explains the somewhat larger error bars. The resulting characteristic parameters are listed in Table I, together with corresponding numbers from our previous work [9]. Averaged values from these six measurements are given as well. They were obtained for the average multiplicity and the average total $\gamma$-ray energy by weighting the individual values with their respective statistical uncertainties according to Table II. Hence, the uncertainties of those mean values consist of a statistical part, calculated from the statistical uncertainties from the six measurements, to which a systematic part was added, corresponding to the mean value of the individual systematic uncertainties. The mean value of the average photon energy is

TABLE II. Relative contributions to the total uncertainties of prompt fission $\gamma$-ray characteristics from our measurements as given in Table I.

\begin{tabular}{lcrr}
\hline \hline Type of uncertainty & $\bar{v}_{\gamma}$ & $\bar{E}_{\gamma, \text { tot }}$ & $\bar{\epsilon}_{\gamma}$ \\
\hline $\begin{array}{l}\text { Statistical ( } \gamma \text {-rays, fissions, } \\
\text { number of simulations) }\end{array}$ & $13.4 \%$ & $8.7 \%$ & $10.2 \%$ \\
Systematic & $86.6 \%$ & $91.3 \%$ & $89.8 \%$ \\
- Simulation (setup, cross sections) & $56.2 \%$ & $54.8 \%$ & $54.9 \%$ \\
- Energy calibration & - & $4.6 \%$ & $3.8 \%$ \\
- Fitting detector response & $30.4 \%$ & $31.9 \%$ & $31.1 \%$ \\
\hline \hline
\end{tabular}

then calculated from the ratio of the averaged values for multiplicity and total energy with their corresponding uncertainties. Our results are compared to results from previous experiments $[2,17,18]$, model calculations $[5,6,8,14,15]$, and the recent evaluation [16]. The last line corresponds to modified values from Ref. [17] according to a procedure explained below. A survey of results is also given in Fig. 2, where the full (red) circles denote the values from this work as well as from our previous one [9], while the open black circles indicate the other available results. Mean values and their uncertainties, based on our measurements, are indicated by full-drawn and dashed (red) lines, respectively. The good repeatability of the technique applied in our experiments is documented by the excellent agreement between the results obtained in each of the six measurements.

\section{DISCUSSION AND CONCLUSIONS}

From both presentations, Table I and Fig. 2, it is obvious that all our measurements, performed independently with high statistics and with different detectors, led to very consistent results. Averaging these gives very precise mean values, i.e., with very small uncertainties. Our results are also in good agreement with those from experiments from the early 1970s $[2,18]$, recent model calculations [14,15], and the evaluated library ENDF/B-VII.1 [16]. The average photon multiplicity from Ref. [17], determined by means of a multiplicity distribution measured with the DANCE detector system, agrees also quite well with our result. This is also reflected in Fig. 3, which shows the measured distribution from Ref. [17] as open black dots and a calculated one from this work as (red) histogram. 


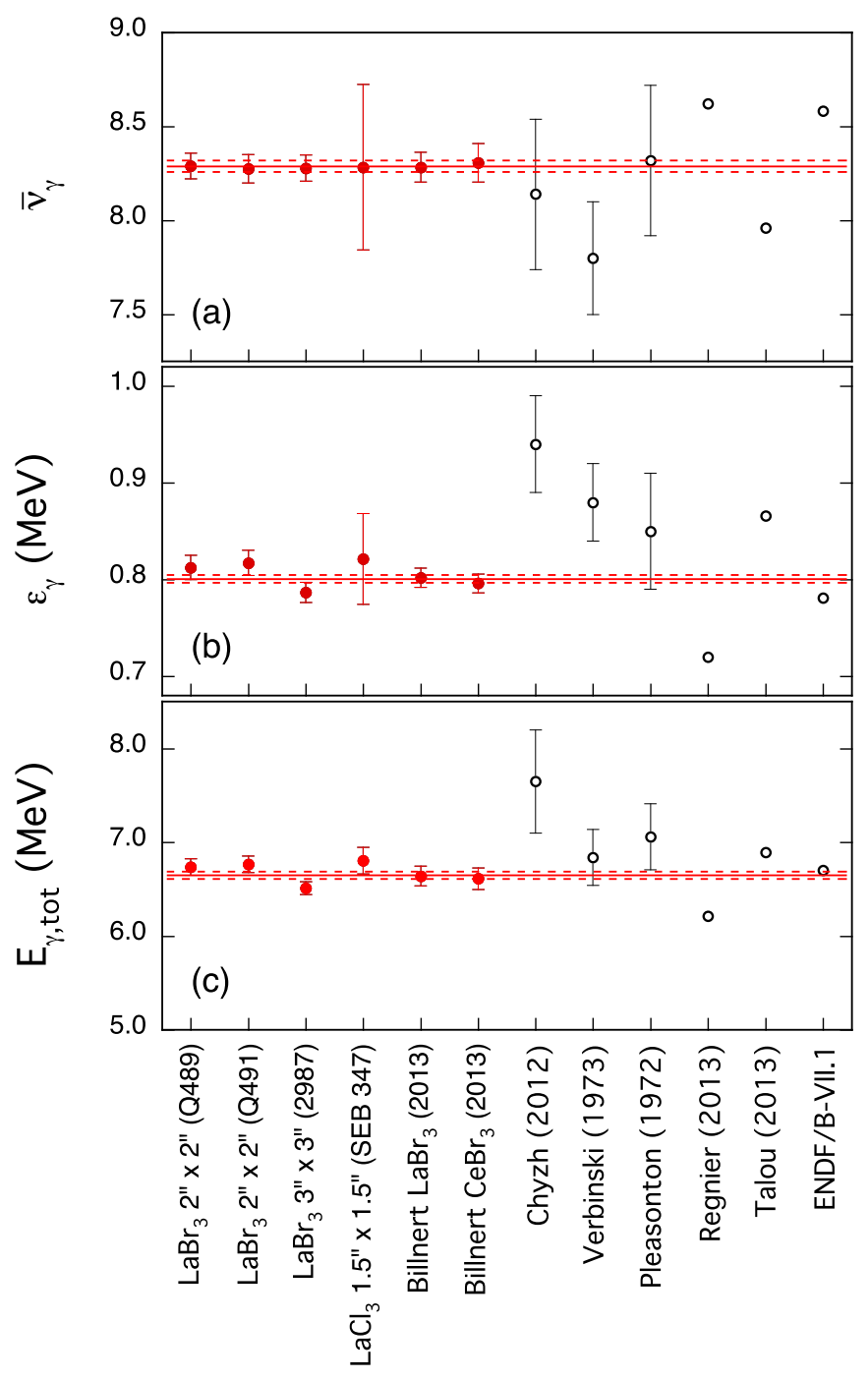

FIG. 2. (Color online) Overview of results for the measurement of prompt $\gamma$-ray emission from the spontaneous fission of ${ }^{252} \mathrm{Cf}$ : (a) Average photon multiplicity, (b) mean photon energy per fission, and (c) total released photon energy. Values from both this work and our previous work [9] are shown in full (red) circles and compared to other experimental results from the early 1970s [2,18] and from a recent work by Chyzh et al. [17]. Also shown are data from ENDF/BVII.1 [16] and results from recent Monte Carlo Hauser-Feshbach calculations $[14,15]$. Values averaged over our results obtained in six measurements with five different detectors and their uncertainties are displayed as full drawn and dashed (red) lines, respectively.

Here we have used a negative binomial distribution to describe the probability $P\left(v_{\gamma}\right)$ for a certain multiplicity $v_{\gamma}$, which may be written as

$$
P\left(v_{\gamma}\right)=\left(\begin{array}{c}
\alpha+v_{\gamma}-1 \\
v_{\gamma}
\end{array}\right) p^{\alpha}(1-p)^{v_{\gamma}},
$$

with $\alpha=\left(\mathrm{D}_{\gamma}-1\right)^{-1}$ and $\mathrm{p}=\alpha /\left(\alpha+\bar{v}_{\gamma}\right)$ [20]. The only parameters to be used here are $\mathrm{D}_{\gamma}=1.074$ according to Ref. [20] and the average $\gamma$-ray multiplicity $\bar{v}_{\gamma}=8.29$ from our work (cf. Table I). The good agreement between both distributions corresponds not only to the good agreement

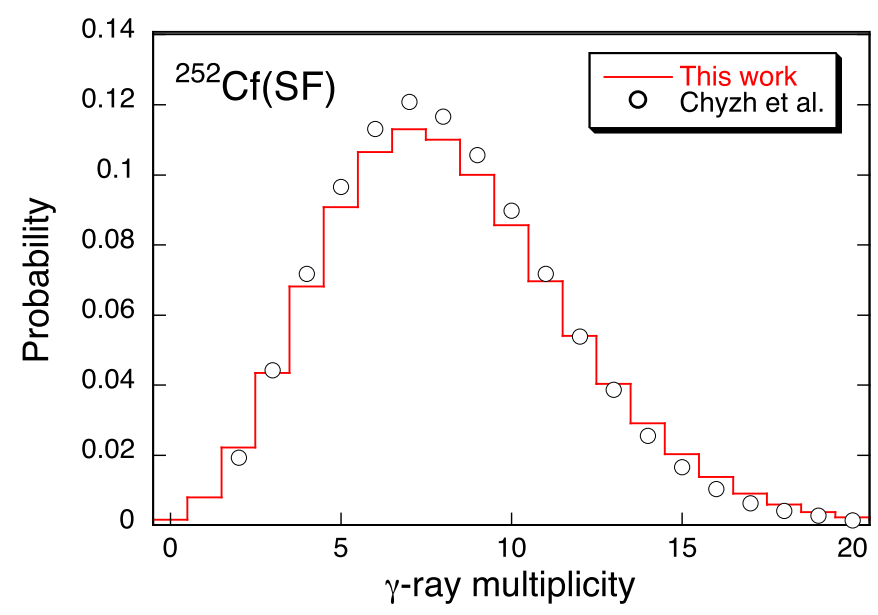

FIG. 3. (Color online) Comparison of prompt fission $\gamma$-ray multiplicity distributions: as (red) histogram the calculated one from this work based on the average experimental value and a negative binomial distribution, and the measured one from Ref. [17] as open black dots.

between both average multiplicities, but it proves also that both-complementary-methods of determining an average multiplicity, i.e., integrating a correctly measured PFGS on one hand and calculating the first moment of a measured multiplicity distribution on the other, give equivalent and consistent results. Moreover, it indicates also the goodness of approximating prompt fission $\gamma$-ray multiplicity distributions with the here-chosen parametrization. This model is also used in the fission module provided by Ref. [21].

In contrast to what we have just shown, the PFGS from DANCE does not result in a comparable average multiplicity, since a big part of photon yield is missing in the spectrum, which is depicted in Fig. 4. Here we have compared the PFGS taken with a $\mathrm{LaBr}_{3}$ :Ce detector from this work with the one obtained by the DANCE collaboration [17], which has been normalized to the spectrum from Ref. [2], as shown in

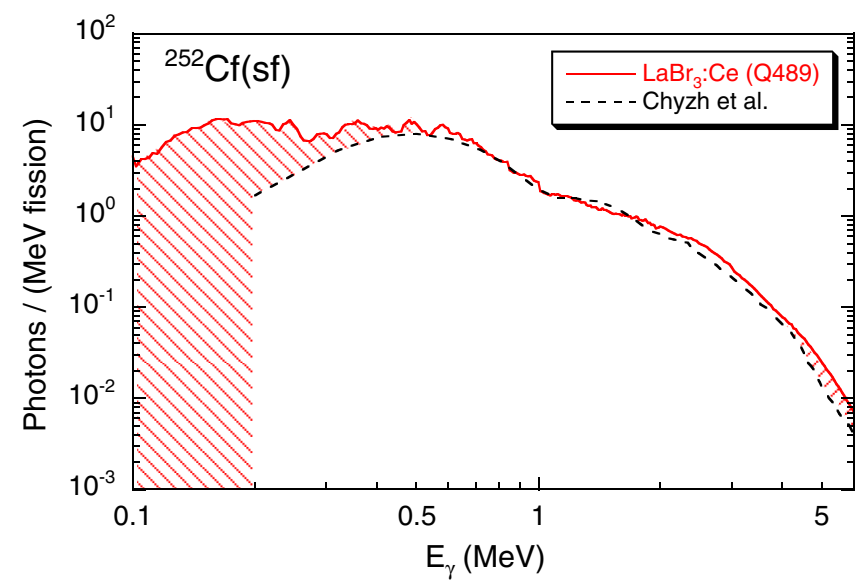

FIG. 4. (Color online) Comparison of PFGS from ${ }^{252} \mathrm{Cf}(\mathrm{sf})$, obtained with one of the $\mathrm{LaBr}_{3}$ :Ce detectors and the one obtained with the DANCE detector system [17], depicted as full (red) and dashed (black) lines, respectively. The (red) hatched areas below $500 \mathrm{keV}$ and above $4 \mathrm{MeV}$ indicate the missing photon yield in the DANCE spectrum (see text for details). 
Ref. [9]. The (red) hatched areas below $500 \mathrm{keV}$ and above $4 \mathrm{MeV}$ indicate the difference in photon yield, which affects practically the low-energy region only and amounts to about $30 \%$, i.e., integration of the spectrum from Ref. [17] gives an average multiplicity of 5.81 compared to our averaged value of 8.29. Please observe that the value of 8.14, as given in Ref. [17] and listed in Table I, has been verified by summing over their multiplicity distribution. In contrast, the mean energy per photon is in accordance with the PFGS shown there. Hence, it is not really a surprise that both average photon energy $\epsilon_{\gamma}$ and total released $\gamma$-ray energy $E_{\gamma \text {,tot }}$ published in Ref. [17] are significantly higher than all other values available so far (see Figs. 2(b) and 2(c), respectively), since a lack of low-energy photons shifts the mean energy toward higher values. We have assessed this effect and present a correction factor for the average photon energy obtained with DANCE.

Since our PFGS and the one from Ref. [17] agree well between $500 \mathrm{keV}$ and $4 \mathrm{MeV}$ (cf. Fig. 4), we calculated the average photon energy for both spectra within this energy region as well as for the entire energy regime. From the ratios of both values for each spectrum we determined a factor of $(0.81 \pm 0.07)$, with which the average photon energy given in Ref. [17] must be multiplied. As a result one obtains $\epsilon_{\gamma}=$ $(0.76 \pm 0.08) \mathrm{MeV}$, which is in good agreement with all the other values depicted in Fig. 2(b). If we also correct the spectrum from Ref. [17] for the loss of photons according to Fig. 4 and compute the average photon multiplicity, we obtain $\bar{v}_{\gamma}=(8.40 \pm 0.81)$, which agrees well with the original value of $(8.14 \pm 0.40)$ deduced from the multiplicity distribution in Ref. [17]. Using both $\epsilon_{\gamma}=(0.76 \pm 0.08) \mathrm{MeV}$ and $\bar{v}_{\gamma}=(8.40 \pm 0.81)$ we may calculate $E_{\gamma, \text { tot }}=(6.38 \pm$ $0.91)$, which also compares well with all other results. These values have been included in Table I (bottom line). The given uncertainties were estimated from the ones given in Refs. [9,17] under the assumption that the relative errors in energy are negligible. It must be noted here that, apart from the multiplicity distribution depicted in Fig. 3, the mean total energy as a function of $\gamma$-ray multiplicity is given in Ref. [17], too. If both pieces of information are combined, one obtains an average total $\gamma$-ray energy of $6.69 \mathrm{MeV}$ and, together with an average multiplicity of 8.14 deduced from Fig. 3, an average energy per photon of $0.82 \mathrm{MeV}$. These values are in excellent agreement with our results as well as with the ones from our corrections to the PFGS from Ref. [17].

We conclude that the determination of the average prompt fission $\gamma$-ray multiplicity, either from a properly measured spectrum or a measured multiplicity distribution, gives equivalent and consistent results, as it ought to be. However, if the measured photon spectrum is not correct, e.g., the low-energy part is underestimated, not only the deduced multiplicity is affected, but also the average energy per photon and the total released $\gamma$-ray energy. Hence, a good knowledge of the PFGS, in particular the low-energy part below $500 \mathrm{keV}$, is crucial for the determination of PFGS characteristics.

\section{ACKNOWLEDGMENT}

One of the authors (R.B.) is indebted to the European Commission for providing support as a PhD fellow at the EC-JRC IRMM, during which this work was carried out.
[1] A. Oberstedt, T. Belgya, R. Billnert, R. Borcea, T. Bryś, W. Geerts, A. Göök, F.-J. Hambsch, Z. Kis, T. Martinez, S. Oberstedt, L. Szentmiklosi, K. Takàcs, and M. Vidali, Phys. Rev. C 87, 051602(R) (2013).

[2] V. V. Verbinski, H. Weber, and R. E. Sund, Phys. Rev. C 7, 1173 (1973).

[3] F. Pleasonton, R. L. Ferguson, and H. W. Schmitt, Phys. Rev. C 6, 1023 (1972).

[4] R. W. Peelle and F. C. Maienschein, Phys. Rev. C 3, 373 (1971).

[5] O. Litaize and O. Serot, Phys. Rev. C 82, 054616 (2010).

[6] D. Regnier, O. Litaize, and O. Serot, Proc. Theory-2 Scientific Workshop on Nuclear Fission Dynamics and the Emission of Prompt Neutron and Gamma Rays, November 2012, Biarritz, France, Phys. Procedia 47 (2013) 47.

[7] P. Talou, T. Kawano, and I. Stetcu, Phys. Procedia 47, 39 (2013).

[8] B. Becker, P. Talou, T. Kawano, Y. Danon, and I. Stetcu, Phys. Rev. C 87, 014617 (2013).

[9] R. Billnert, F.-J. Hambsch, A. Oberstedt, and S. Oberstedt, Phys. Rev. C 87, 024601 (2013).

[10] S. Oberstedt, R. Billnert, T. Belgya, T. Bryś, W. Geerts, C. Guerrero, F.-J. Hambsch, Z. Kis, A. Moens, A. Oberstedt, G. Sibbens, L. Szentmiklosi, D. Vanleeuw, and M. Vidali, Phys. Rev. C 90, 024618 (2014).
[11] A. Oberstedt, S. Oberstedt, R. Billnert, W. Geerts, F.-J. Hambsch, and J. Karlsson, Nucl. Instrum. Methods Phys. Res., Sect. A 668, 14 (2012).

[12] S. Oberstedt, R. Borcea, T. Brys, Th. Gamboni, W. Geerts, F.-J. Hambsch, A. Oberstedt, and M. Vidali, Nucl. Instrum. Methods Phys. Res., Sect. A 714, 31 (2013).

[13] http://www.oecd-nea.org/tools/abstract/detail/nea-1525.

[14] D. Regnier (private communication)

[15] P. Talou (private communication).

[16] ENDF/B-VII.1 Evaluated Nuclear Data File ZA $=98251, \mathrm{MF}=$ 15, MT = 18 (2011), http://www.nndc.bnl.gov/exfor/endf00.jsp.

[17] A. Chyzh, C. Y. Wu, E. Kwan, R. A. Henderson, J. M. Gostic, T. A. Bredeweg, R. C. Haight, A. C. Hayes-Sterbenz, M. Jandel, J. M. O’Donnell, and J. L. Ullman, Phys. Rev. C 85, 021601(R) (2012).

[18] F. Pleasonton, R. L. Ferguson, and H. W. Schmitt, Oak Ridge National Laboratory, ORNL-4844, 109-112; in T. E. Valentine, Ann. Nucl. Energy 28, 191 (2001).

[19] M. Heil, R. Reifarth, M. M. Fowler, R. C. Haight, F. Käppeler, R. S. Rundberg, E. H. Seabury, J. L. Ullmann, J. B. Wilhelmy, and K. Wisshak, Nucl. Instrum. Methods Phys. Res., Sect. A 459, 229 (2001).

[20] T. E. Valentine, Ann. Nucl. Energy 28, 191 (2001).

[21] J. M. Verbeke, C. Hagmann, and D. Wright, Lawrence Livermore National Laboratory, UCRL-AR-228518, May 11, 2010 . 